\title{
Duración subóptima del sueño y fenotipo circadiano en mujeres adultas residentes de Valencia, Venezuela atendidas en jornadas de salud. Perfil cardiometabólico asociado
}

Nelina Ruiz-Fernández* 1,4,5; Doris Nobrega ${ }^{1,6}$; Indira Varela ${ }^{1,4}$; Yolima Fernández ${ }^{1,7}$; Claudia Mendoza ${ }^{1,8}$; Jhon Jesus ${ }^{2,9}$; Gabriel Villalobos ${ }^{3}$; Cesar Vega ${ }^{3}$; María Yoris ${ }^{3}$; Dessire Zamora ${ }^{3}$

\section{RESUMEN}

Objetivo: Evaluar la duración del sueño y fenotipos circadianos y su asociación con variables sociodemográficas y clínicas, de estilo de vida e indicadores de riesgo cardiometabólico en mujeres adultas.

Materiales y métodos: Estudio transversal de 108 mujeres adultas residentes de la ciudad de Valencia, Venezuela atendidas en jornadas de salud. Se establecieron variables sociodemográficas, clínicas y estilo de vida, duración de sueño autoinformada durante la semana, fin de semana y ponderada (corta: $<7 \mathrm{~h}$ o larga: $>9 \mathrm{~h}$ ) y fenotipo circadiano o cronotipo mediante la versión reducida del cuestionario de matutinidad de Horne y Östberg. Se midieron presión arterial, peso, talla, circunferencia de cintura y biomarcadores en sangre venosa. Se calcularon tres puntajes de estatus cardiometabólico. Se estableció hipertensión arterial (HTA) y síndrome metabólico (SM). Resultados: Se encontró duración corta de sueño ponderada en 21,3 \% de las mujeres y duración larga ponderada en $16,7 \%$. El 28,7 \% mostró cronotipo claramente matutino; 38 \%, moderadamente matutino; $32,4 \%$, indefinido y $0,9 \%$, moderadamente vespertino, sin casos de cronotipo claramente vespertino. La duración corta de sueño y los cronotipos matutinos fueron más frecuentes en mujeres mayores de 40 años. La duración de sueño se asoció a estado civil, ocupación y condición de exfumadora; los cronotipos se asociaron a posmenopausia. La duración corta se asoció a obesidad abdominal e hipertensión arterial (HTA), elevación de gamma-glutamil transferasa y ácido úrico. La duración larga se asoció a glicemia y transaminasas más elevadas. El cronotipo claramente matutino se asoció a HTA, SM y número de componentes del SM presentes. La duración corta de sueño y el cronotipo claramente matutino predijeron elevado riesgo cardiometabólico, al ajustar por edad y posmenopausia esta asociación desapareció.

Conclusiones: La duración subóptima del sueño y los fenotipos circadianos se asociaron a variables socio demográficas, clínicas y de riesgo cardiometabólico. La duración corta de sueño y el cronotipo claramente matutino predijeron elevado riesgo cardiometabólico.

Palabras clave: Sueño; Privación de sueño; Ritmo circadiano; Enfermedades cardiovasculares; Síndrome metabólico (Fuente: DeCS BIREME).

\section{Suboptimal sleep duration and circadian phenotype in adult women residents of Valencia, Venezuela, treated in health campaigns: an associated cardiometabolic profile}

\section{ABSTRACT}

Objective: To evaluate the sleep duration, the circadian phenotypes, and their association with sociodemographic and clinical variables, lifestyle, and indicators of cardiometabolic risk in adult women.

Materials and methods: A cross-sectional study of 108 adult women living in the city of Valencia, Venezuela, who were treated in health campaigns. Sociodemographic and clinical variables, lifestyle, self-reported sleep duration during weekdays and weekends, and weighted sleep duration (short: < 7 hours or long: >9 hours) were determined. The circadian phenotype or chronotype were assessed by the reduced Horne \& Östberg's morningness-eveningness questionnaire. Blood pressure, weight, height, waist circumference, and blood biomarkers were measured. Three (3) cardiometabolic status scores were calculated. Hypertension (HTN) and metabolic syndrome (MS) were established. Results: Twenty one point three percent $(21.3 \%)$ of the study population presented a short weighted sleep duration and $16.7 \%$ presented a long one. Twenty-eight point seven percent $(28.7 \%)$ showed a clearly morning chronotype, $38 \%$ showed a moderately morning chronotype, $32.4 \%$ showed no definite chronotype, $0.9 \%$ showed a moderately evening chronotype, and there were no cases of clearly evening chronotype. Short sleep duration and morning chronotypes were more frequent in women $>40$ years. The sleep duration was associated with marital status, 
Duración subóptima del sueño y fenotipo circadiano en mujeres adultas residentes de Valencia, Venezuela atendidas en jornadas de salud. Perfil cardiometabólico asociado

occupation and ex-smoker status. The chronotypes were associated with postmenopause. The short sleep duration was associated with abdominal obesity and HTN, and elevation of gamma-glutamyltransferase and uric acid. The long sleep duration was associated with higher glycemia and transaminases. The clearly morning chronotype was associated with HTN, MS and presence of a number of MS components. The short sleep duration and the clearly morning chronotype predicted a high cardiometabolic risk. This association disappeared by adjusting the age and postmenopause status.

Conclusions: The suboptimal sleep duration and the circadian phenotypes were associated with sociodemographic, clinical and cardiometabolic risk variables. The short sleep duration and the clearly morning chronotype predicted a high cardiometabolic risk.

Keywords: Sleep; Sleep deprivation; Circadian rhythm; Cardiovascular diseases; Metabolic syndrome (Source: MeSH NLM).

1. Universidad de Carabobo, Facultad de Ciencias de la Salud, Escuela de Bioanálisis, Sede Carabobo. Carabobo, Venezuela.

2. Universidad de Carabobo, Facultad de Ciencias de la Salud, Escuela de Ciencias Biomédicas y Tecnológicas. Carabobo, Venezuela.

3. Universidad de Carabobo, Facultad de Ciencias de la Salud, Escuela de Medicina, Sede Carabobo. Carabobo, Venezuela.

4. Universidad de Carabobo, Facultad de Ciencias de la Salud, Departamento de Morfofisiopatología. Carabobo, Venezuela.

5. Universidad de Carabobo, Facultad de Ciencias de la Salud, Instituto de Investigaciones en Nutrición (INVESNUT). Carabobo, Venezuela.

6. Universidad de Carabobo, Facultad de Ciencias de la Salud, Departamento de Ciencias Básicas. Carabobo, Venezuela.

7. Universidad de Carabobo, Facultad de Ciencias de la Salud, Departamento Investigación y Desarrollo Profesional. Carabobo, Venezuela.

8. Universidad de Carabobo, Facultad de Ciencias de la Salud, Departamento de Estudios Clínicos. Carabobo, Venezuela.

9. Universidad de Carabobo, Facultad de Ciencias de la Salud, Departamento de Parasitología. Carabobo, Venezuela.

*Autor corresponsal 


\section{INTRODUCCIÓN}

Según el Informe del Estado de las Enfermedades Crónicas No Transmisibles (ECNs) publicado por la Organización Mundial de la Salud (OMS) en 2014, las enfermedades cardiovasculares constituyeron la principal causa de muerte, ya que representaron el $46 \%$ del total de las muertes por ECNs, mientras que la diabetes explicó $4 \%$ de los decesos ${ }^{(1)}$. La revisión de los Anuarios de Mortalidad correspondientes a una década muestra que el mismo perfil se repite en Venezuela, ya que son precisamente las ECNs relacionadas a riesgo cardiometabólico $(\mathrm{RCM})$ las que más defunciones originaron en el país. En 2013, último año para el cual se encuentran disponibles las cifras de mortalidad, las enfermedades del corazón, la diabetes mellitus y las enfermedades cerebro-vasculares ocuparon la primera, tercera y cuarta causa de mortalidad global en Venezuela, respectivamente (2). Cuando se revisa la distribución por género se observa que, entre los hombres, esas enfermedades fueron la primera, la quinta y la sexta causas de muerte, mientras que para el sexo femenino representaron la primera, tercera y quinta causa. En conjunto, los datos estadísticos antes referidos apuntan hacia la necesidad de desarrollar, en las mujeres venezolanas, investigaciones focalizadas en el RCM, un término que en los últimos años se aplica a la probabilidad de desarrollar enfermedades cardiovasculares y diabetes mellitus.

El sueño parece ser la única "actividad sedentaria" que podría proteger contra las enfermedades cardiometabólicas, ya que no solo cumple una función restaurativa sino también regulatoria como un importante modulador de los procesos neuroendocrinos y del metabolismo energético ${ }^{(3)}$. Estudios transversales y longitudinales en adultos han demostrado que la duración subóptima del sueño, tanto corta (generalmente $<6$ horas) como larga (generalmente > 8 horas), se asocia con obesidad, hipertensión arterial y diabetes ${ }^{(4,5)}$. Un metaanálisis ${ }^{(6)}$ reveló una asociación significativa entre duración corta de sueño y riesgo de síndrome metabólico (SM).

El sueño está estrechamente vinculado a los ritmos internos del cuerpo o ritmos circadianos. Los ritmos circadianos son fundamentales para la salud ya que tienen efectos prominentes sobre la homeostasis endocrina y metabólica (7). En cada individuo existe una propensión o preferencia específica a dormir en un momento determinado del periodo de 24 horas, lo cual es conocido como cronotipo o fenotipo circadiano, su caracterización permite entender la organización de los procesos regulatorios. El cronotipo es un atributo de los seres humanos que refleja la fase circadiana individual ${ }^{(8)}$. Las diferencias de fase se manifiestan en distintas preferencias entre individuos, de este modo, existen individuos que concentran más su energía y eficiencia en las primeras horas de la mañana, luego de un despertar temprano, o sujetos que, por el contrario, focalizan sus recursos mentales y físicos hacia la media tarde y hasta entrada la noche, después de despertar ya entrada la mañana. Los primeros exhiben un cronotipo "matutino" y los últimos el cronotipo "vespertino", que representan los extremos de la preferencia. La mayoría de individuos se integran principalmente al denominado cronotipo "intermedio", es decir, son flexibles en su preferencia por dormir en un momento determinado de las 24 horas del día ${ }^{(9)}$.

Existen evidencias de que el cronotipo pudiera explicar parte del RCM que desarrollan ciertos individuos. En el estudio de Yu et al. ${ }^{(10)}$ el cronotipo vespertino se asoció a diabetes y sarcopenia en hombres y a SM en mujeres, independientemente de la duración del sueño y otros problemas de salud. En comparación con otros cronotipos, las mujeres vespertinas mostraron mayor circunferencia de cintura y masa grasa corporal (visceral y subcutánea) así como concentraciones más elevadas de triglicéridos y proteína C-reactiva.

El impacto de la duración del sueño sobre el RCM está poco documentado en el contexto venezolano. En un intento por aportar datos, Querales et al. (11) informaron que la insuficiencia de sueño (registrada como 14 o más días de sueño o descanso insuficiente en el último mes) fue frecuente en un grupo de 85 mujeres adultas del estado Carabobo, Venezuela; además, la insuficiencia del sueño se encontró asociada significativamente a edad mayor de 40 años, exceso de peso, obesidad central, hipertrigliceridemia, relación colesterol total/HDLc elevada, relación triglicéridos/HDLc elevada y mayor riesgo de SM. Por su parte, al estudiarse la prevalencia de síntomas relacionados con el sueño en cuatro ciudades latinoamericanas (Ciudad de México, Montevideo, Santiago de Chile y Caracas) se encontró que los ronquidos, el insomnio y la somnolencia diurna mostraron frecuencias de $60,2 \%, 34,7 \%$ y $16,4 \%$, respectivamente ${ }^{(12)}$; dicho trabajo no estuvo dirigido a demostrar asociaciones entre los síntomas estudiados y el RCM.

En nuestro conocimiento, en Venezuela existe un vacío de información sobre la caracterización de los fenotipos circadianos, en un aspecto general y, específicamente, respecto a su posible asociación con el RCM. En Latinoamérica, trabajos previos en Colombia ${ }^{(13)}$ y Brasil (14) han demostrado orientación matutina en un importante porcentaje de los individuos adultos estudiados, lo que contrasta con hallazgos de Norteamérica y países del norte del Europa. Estas investigaciones no han incluido el RCM como variable de estudio.

Las anteriores consideraciones justificaron la formulación del presente estudio, que se propuso evaluar la duración del sueño y fenotipos circadianos y su asociación con diversas variables sociodemográficas y clínicas, factores 
de estilo de vida e indicadores de riesgo cardiometabólico en un grupo de mujeres adultas residentes de la ciudad de Valencia, Venezuela, atendidas en jornadas de salud.

\section{MATERIALES Y MÉTODOS}

\section{Diseño y población del estudio}

Es un estudio descriptivo correlacional, de tipo transversal, de campo y no experimental. La población estuvo constituida por todas aquellas mujeres adultas que asistieron a sesiones de despistaje de factores de riesgo cardiovascular $(\mathrm{N}=128)$ ejecutadas en el marco de jornadas de atención de salud organizadas por la Iglesia Adventista del Séptimo Día, en el municipio Valencia de la ciudad de Valencia (N10 ${ }^{\circ}$ '43.27"), estado Carabobo-Venezuela, entre julio 2015 y diciembre 2016. Para la selección de la muestra se aplicó un muestreo no probabilístico de tipo intencional (por conveniencia), en el que se seleccionaron 108 mujeres que cumplieron con los criterios de inclusión que fueron tener entre 20 y 65 años de edad, ser aparentemente sana, sin síntomas de procesos infecciosos/inflamatorios, residentes de la ciudad de Valencia, y que completaron todas las evaluaciones previstas. Se excluyó del estudio toda mujer adulta que presentara uno o más de las siguientes condiciones: antecedente personal de enfermedad cardiovascular, hipertensión no controlada, diagnóstico de cáncer, diabetes, insuficiencia renal o hepática, enfermedades autoinmunes o inflamatorias crónicas (artritis reumatoide o espondilitis anquilosante), depresión, discapacidad intelectual, enfermedad tiroidea o suprarrenal, cirugía o trauma mayor reciente, estar en tratamiento con insulina, corticoides o psicotrópicos así como terapia de reemplazo hormonal, en un régimen voluntario de pérdida de peso, actividad laboral con turnos rotativos diurnos/nocturnos, embarazo y lactancia.

\section{Variables y mediciones}

Un investigador previamente entrenado aplicó a cada participante un instrumento de recolección para obtener datos personales y sociodemográficos (estado civil y ocupación actual), antecedentes personales y familiares de enfermedades cardiometabólicas (en grado de padre y madre) y de otras condiciones consideradas como criterios de exclusión, hábito tabáquico (sí/no), hábito de ingesta de alcohol (sí/no) y su frecuencia, fecha de última menstruación y tratamiento farmacológico. La ocupación se categorizó en estudiante, trabajador manual, trabajador de oficina, profesional y ama de casa.

En forma retrospectiva, por recuerdo, las participantes indicaron la hora de acostarse a dormir en la noche y la de despertarse cada uno de los siete días de la semana. Esta información permitió conocer la duración del sueño durante la semana (DSS) y el fin de semana (DSFS); para este análisis se consideró como fin de semana desde la noche del viernes hasta la mañana del domingo. Adicionalmente, se calculó la duración de sueño ponderada de la semana (DSP), así: [(número promedio de horas de sueño durante la semana $\times 5$ ) + (número promedio de horas de sueño durante fin de semana $\times 2$ )]/7. Se definió la duración subóptima del sueño según las recomendaciones actualizads de la Fundación Nacional del Sueño (15), de modo tal que DSS, DSFS y DSP se categorizaron como corta cuando fueron $<7 \mathrm{~h}$ y larga cuando fueron $>9 \mathrm{~h}$.

El fenotipo circadiano o cronotipo se determinó a través de la versión española estandarizada de la escala reducida del cuestionario de matutinidad (MEQr) de Horne y Östberg, construida por Adán y Almirall (16). Comprende cinco preguntas basadas en la versión original del MEQ. Las preguntas 1 a 3 piden indicar la hora del día cuando el individuo se siente en su mejor momento, el momento en que prefiere levantarse, y en el que prefiere ir a la cama. La pregunta 4 se relaciona con el grado de cansancio percibido en la primera media hora después de despertar. Por último, en la pregunta 5, se pide indicar su preferencia por la matutinidad o vespertinidad. El cuestionario reducido ha demostrado fiabilidad, con una adecuada correlación inter-ítem y validez (8). Para la obtención del cronotipo se sumó la puntuación de cada ítem del MEQr y el puntaje total se comparó con la siguiente escala: $22-25$ puntos, tipo claramente matutino; 18-21 puntos, tipo moderadamente matutino; $12-17$ puntos, tipo indefinido; 8-11, tipo moderadamente vespertino; 4-7 puntos, tipo claramente vespertino ${ }^{(16)}$.

Se efectuaron medidas antropométricas y de presión arterial, así como la determinación en sangre de biomarcadores de orden metabólico, hepático y proinflamatorio / protrombótico. Las medidas antropométricas se realizaron sin zapatos y con ropa mínima. Personal entrenado para tal fin determinó el peso con una balanza (HealthMeter) previamente calibrada (precisión $=0,1 \mathrm{~g}$ ) y la talla con una cinta métrica no extensible (precisión $=0,1 \mathrm{~cm}$ ) adosada a la pared, y se aplicaron protocolos estándares. Para medir la circunferencia de cintura (CC) se colocó una cinta métrica de igual precisión a la altura del punto medio entre la última costilla y la cresta ilíaca, con el sujeto en bipedestación al final de la espiración no forzada. Se calculó el índice de masa corporal (IMC- $\mathrm{kg} / \mathrm{m} 2$ ) para establecer exceso de peso (sobrepeso y obesidad), y el índice cintura/talla (CC/T) para establecer obesidad abdominal, de acuerdo a los criterios propuestos por la OMS ${ }^{(17)}$ y por Ashwell y Hsieh ${ }^{(18)}$, respectivamente.

La presión arterial se determinó con un esfigmomanómetro de mercurio calibrado, se aplicó el método auscultatorio y se siguieron las recomendaciones americanas. Cifras de presión sistólica $>140 \mathrm{~mm} \mathrm{Hg}$ y/o de presión diastólica $>90 \mathrm{~mm} \mathrm{Hg}$ para el momento del examen o cuando el individuo refirió tratamiento hipotensor ${ }^{(19)}$ definieron el diagnóstico de hipertensión arterial (HTA).

Personal debidamente entrenado realizó una venopunción 
en el pliegue del codo y se separaron alícuotas de plasma y suero por centrifugación. Se valoró la concentración de fibrinógeno en el plasma mediante el método gravimétrico descrito por Ingram ${ }^{(20)}$. En suero se determinaron glucosa, colesterol total (CT), triglicéridos (TGL) y ácido úrico, a través de kits comerciales fundamentados en métodos enzimático-colorimétricos de rutina; el colesterol unido a HDL (HDLc) se midió previa precipitación con reactivo de fosfotungstato. Se estimó el colesterol unido a LDL (LDLC) mediante la fórmula de Friedewald. La actividad en suero de la gamma-glutamiltransferasa (GGT) y de las transaminasas glutámico oxalacética (TGO) y pirúvica (TGP) se determinaron mediante kits comerciales basados en métodos cinéticos rutinarios. Todas las mediciones en suero se realizaron con el empleo de un espectofotómetro OMEGA IV.

Se calcularon las relaciones TGO/TGP, CT/HDLc, LDLc/ HDLc, TGL/HDLc y colesterol NoHDL (CT-HDLc). La presencia de SM y la categorización de sus componentes individuales se determinaron según definición armonizada (21). Se estableció estado proinflamatorio/protrombótico cuando la concentración de fibrinógeno fue $\geq 321 \mathrm{~g} / \mathrm{L}^{(22)}$.

Adicionalmente, se calcularon puntajes o scores continuos del estatus cardiometabólico: 1. Índice cardiometabólico (ICMet), fue propuesto por Wakabayashi y Daimon ${ }^{(23)}$ y se calcula como el producto de la relación TGL/HDLc y el índice $C C / T$. 2. siMS score y siMS risk score (por sus siglas originales en inglés), el primero de ellos fue propuesto para la evaluación del estatus del SM y el segundo para evaluar el riesgo de enfermedades coronarias o eventos cerebrovasculares. siMS score se calcula a partir del índice $\mathrm{CC} / \mathrm{T}$, glicemia, TGL, presión arterial sistólica y HDLC, considerando los puntos de corte empleados para definición de SM en el caso de las cuatro últimas variables nombradas (24), siMS risk score incorpora la edad y el antecedente familiar de evento cardio o cerebrovascular a los mismos elementos de siMS score ${ }^{(24)}$. Estos scores se obtuvieron con ayuda de la hoja de cálculo suministrada por Soldatovic et al. (24), y se introdujeron los puntos de corte de la definición de SM que se aplicó en la presente investigación. Ya que por el momento no existen puntos de corte para los puntajes descritos, se calculó el percentil 85 de cada uno. Valores de ICMet, siMS score y siMS risk score iguales o mayores del percentil 85 se consideraron como elevados y las participantes fueron consideradas en alto RCM.

\section{Análisis estadístico}

Se utilizó el software Statistical Package for the Social Sciences (IBM SPSS Statistics, SPSS, Chicago, IL, USA) en su versión 20.0.0 para Windows. El test de KolmogorovSmirnov se empleó para probar si las variables siguieron la distribución normal. Se calcularon estadísticos descriptivos (media, desviación estándar, mediana, rango intercuartílico y frecuencias absolutas y relativas) para caracterizar la muestra estudiada. Las pruebas de $t$ de Student no pareada y U de Mann Whitney, según el caso, permitieron comparar las variables de sueño y cronotipo de acuerdo a grupos etarios (< 40 años y $\geq 40$ años) así como los biomarcadores y puntajes de estatus cardiometabólico según duración de sueño (corta y larga vs. normal) y cronotipo. Se aplicó la prueba de Chi cuadrado para asociar la frecuencia de duración subóptima del sueño y fenotipos circadianos a la edad, estado civil, ocupación, hábito tabáquico y de ingesta de alcohol, condición de posmenopausia, exceso de peso, obesidad abdominal, HTA, SM y número de componentes individuales de este, estado proinflamatorio/protrombótico y alto RCM. Con base a las asociaciones encontradas a través de la prueba de Chi cuadrado, se realizó un análisis de regresión logística para estimar odds ratios (OR) o razones de oportunidad para SM, HTA, obesidad abdominal o alto RCM en asociación a duración subóptima de sueño y cronotipo, con o sin ajuste por edad y posmenopausia. Se empleó el método de selección por pasos hacia delante para la introducción/ remoción de las variables en el modelo de regresión.

\section{Consideraciones éticas}

Esta investigación contó con el aval institucional de la Facultad de Ciencias de la Salud de la Universidad de Carabobo, Venezuela y cumplió con los lineamientos de la Declaración de Helsinki en su versión del año 2013. Se explicaron los objetivos y detalles del estudio a las mujeres participantes, y se respondieron sus preguntas. Luego se solicitó el consentimiento informado por escrito.

\section{RESULTADOS}

\section{Caracterización de la muestra estudiada}

Se evaluó un grupo de 108 mujeres con una edad media de $38 \pm 12,4$ años (rango: 18-63 años). La distribución de acuerdo al estado civil fue $61,1 \%$ eran solteras, 32,2 \%, casadas; $2,8 \%$, divorciadas y $1,9 \%$ vivían en concubinato. En cuanto a ocupación, se identificó como estudiante $11,5 \%$ de la muestra, ama de casa $27,9 \%$, trabajadora de oficina $22,1 \%$, trabajadora manual $19,4 \%$ y profesional $19,4 \%$. Solo cuatro mujeres se identificaron como desempleadas..

Los antecedentes paternos o maternos se distribuyeron en HTA (45,4\%), ACV (13,9\%), enfermedad cardiaca isquémica $(19,4 \%)$ y diabetes $(29,6 \%)$. Además, $4,6 \%$ de las mujeres del estudio declaró ser fumadora, y 8,3\% se calificó como exfumadora. El consumo de bebidas alcohólicas fue reportado por $25 \%$ de las participantes, que lo describieron como eventual o que ocurría una vez a la semana.

El exceso de peso (sobrepeso y obesidad) según IMC fue 41,2 \%; la obesidad según el índice CC/T se observó en $54,5 \%$. 
La HTA se encontró en $25 \%$, SM en $24,7 \%$ y el estado proinflamatorio/ protrombótico se presentó en $44,7 \%$. Se encontró que $15,8 \%$ de las mujeres presentaron ICMet y siMS risk score elevado y $11,8 \%$ siMS score elevado.

Duración subóptima de sueño, cronotipos, variables sociodemográficas y factores de estilo de vida

Al ponderar la semana completa, $21,3 \%$ de las participantes presentaron duración corta de sueño y $16,7 \%$ mostraron duración larga. Los fenotipos circadianos se distribuyeron en claramente matutino $(28,7 \%)$, moderadamente matutino (38\%), indefinido $(32,4 \%)$ y moderadamente vespertino $(0,9 \%)$. Nos se presentaron casos de cronotipo claramente vespertino.

La tabla 1 presenta los indicadores de la duración de sueño y cronotipo en la muestra total y según edad. Entre las mujeres de 40 o más años de edad, la hora de levantarse durante el fin de semana fue significativamente menor, mientras que su puntaje total en el MEQr y los correspondientes a los ítems $1,2,3$ y 5 del mismo instrumento fueron mayores en comparación con las mujeres menores de 40 años.

Tabla 1. Indicadores de la duración de sueño y cronotipo en la muestra total y según edad

\begin{tabular}{|c|c|c|c|}
\hline \multirow[b]{2}{*}{ Variable } & \multirow[b]{2}{*}{ Grupo total $(n=108)$} & \multicolumn{2}{|c|}{ Edad } \\
\hline & & $\begin{array}{c}<40 \text { años } \\
(n=57)\end{array}$ & $\begin{array}{c}\geq 40 \text { años } \\
(n=51)\end{array}$ \\
\hline Hora de acostarse en semana (h:min) & $10: 00(9: 00-10: 00)$ & $9: 48(9: 00-10: 00)$ & $10: 00(9: 00-10: 00)$ \\
\hline Hora de levantarse en semana (h:min) & $5: 24(5: 00-6: 00)$ & $5: 30(5: 00-6: 00)$ & $5: 00(4: 45-6: 00)$ \\
\hline DSS (horas) & $7,70(6,85-8,50)$ & $8,00(7,00-8,55)$ & $7,50(6,60-8,00)$ \\
\hline Hora de acostarse en fin de semana (h:min) & $10: 00(9: 00-10: 23)$ & $10: 00(9: 00-10: 15)$ & $10: 00(9: 00-10: 30)$ \\
\hline Hora de levantarse en fin de semana (h:min) & $6: 37(5: 50-8: 00)$ & $7: 00(6: 00-8: 00)$ & $6: 00(5: 00-7: 15)^{*}$ \\
\hline DSFS (horas) & $8,50(7,81-9,75)$ & $8,75(8,00-10,00)$ & $8,50(7,50-9,25)$ \\
\hline DSP (horas) & $8,00(7,04-8,77)$ & $8,14(7,30-8,89)$ & $7,93(7,00-8,28)$ \\
\hline \multicolumn{4}{|l|}{ Puntajes de los ítems del MEQr } \\
\hline Hora preferida para levantarse (ítem 1) & $4,0(3,0-5,0)$ & $4,0(3,0-5,0)$ & $5,0(3,0-5,0)^{*}$ \\
\hline Condición media hora después de levantarse (ítem 2) & $3,0(2,0-3,0)$ & $3,0(1,0-3,0)$ & $3,0(2,0-4,0)^{\star *}$ \\
\hline Hora de sentirse cansado e irse a dormir (ítem 3) & $5,0(5,0-5,0)$ & $5,0(5,0-5,0)$ & $5,0(5,0-5,0)$ \\
\hline Hora del día en que se encuentra mejor (ítem 4) & $4,0(3,0-5,0)$ & $4,0(3,0-4,0)$ & $4,0(3,0-5,0)^{* *}$ \\
\hline Autoevaluación del cronotipo (ítem 5) & $4,0(4,0-6,0)$ & $4,0(4,0-6,0)$ & $4,0(4,0-6,0)$ \\
\hline Puntaje total del MEQr & $19,0(17,0-22,0)$ & $18,0(16,0-21,0)$ & $20,0(18,0-22,0)^{* *}$ \\
\hline
\end{tabular}

Datos expresados como mediana (rango intercuartílico). Prueba de U de Mann Whitney entre grupos etarios. ${ }^{*} p<0,05 ;{ }^{* *} p<0,01$, entre grupos etarios. DSS: duración de sueño en semana; DSFS: duración de sueño en fin de semana; DSP: duración de sueño ponderada; MEQr: morningness-eveningness questionnarie de escala reducida.

La figura 1A muestra la frecuencia de la duración subóptima (corta y larga) del sueño, durante días de la semana, fin de semana y ponderada, en el grupo total y según grupo etario. La duración corta del sueño fue más frecuente entre las mujeres de 40 o más años, mientras que la duración larga fue de mayor frecuencia entre las mujeres más jóvenes, aunque la asociación no alcanzó significancia estadística. En cuanto a la frecuencia de los fenotipos circadianos, la figura $1 \mathrm{~B}$ muestra que los cronotipos clara y moderadamente matutinos fueron más frecuentes entre las mujeres de 40 o más años $(p=0,017)$.
Al evaluar la asociación de la duración del sueño y del cronotipo con el estado civil, ocupación, postmenopausia, hábito tabáquico y de ingesta de alcohol, se evidenció que la frecuencia de duración larga en fin de semana fue mayor entre las mujeres divorciadas y solteras $(p=0,008)$. Asimismo, la duración corta de sueño en fin de semana fue significativamente asociada a la ocupación de trabajadora manual o ama de casa $(p=0,021)$ y a la condición de exfumadora $(p=0,032)$; también tendió a ser más frecuente entre las mujeres posmenopáusicas pero sin alcanzar significancia estadística. El cronotipo se relacionó a la menopausia, lo que se evidencia al encontrar que la mitad 
de las mujeres posmenopaúsicas presentaron cronotipo claramente matutino $(\mathrm{p}=0,014)$

Duración subóptima de sueño, cronotipos e indicadores antropométricos y bioquímicos de RCM

Se encontraron diferencias significativas en biomarcadores de orden metabólico y hepático cuando se compararon la duración corta o larga de sueño (en la semana, fin de semana y ponderada) con la duración recomendada de 7-9 horas/noche. El índice TGO/TGP fue significativamente menor entre las mujeres que mostraron duración corta del sueño en la semana $(p=0,042)$. La actividad de GGT y la concentración de ácido úrico fueron significativamente mayores entre las mujeres con duración corta del sueño en fin de semana $(p=0,020)$. La glicemia fue significativamente mayor entre las mujeres con duración larga de sueño en la semana ( $p=0,039)$. Las mujeres con duración larga de sueño en fin de semana mostraron valores de glicemia $(p=0,030)$, TGO $(p=0,049)$ y TGP $(p=0,014)$ significativamente mayores $(p=0,003)$. Las mujeres que tuvieron duración larga de sueño ponderada (semana completa) mostraron valores de glicemia $(p=0,016)$ y TGP $(p=0,032)$ significativamente mayores, pero valores menores de GGT $(p=0,044)$.

A)

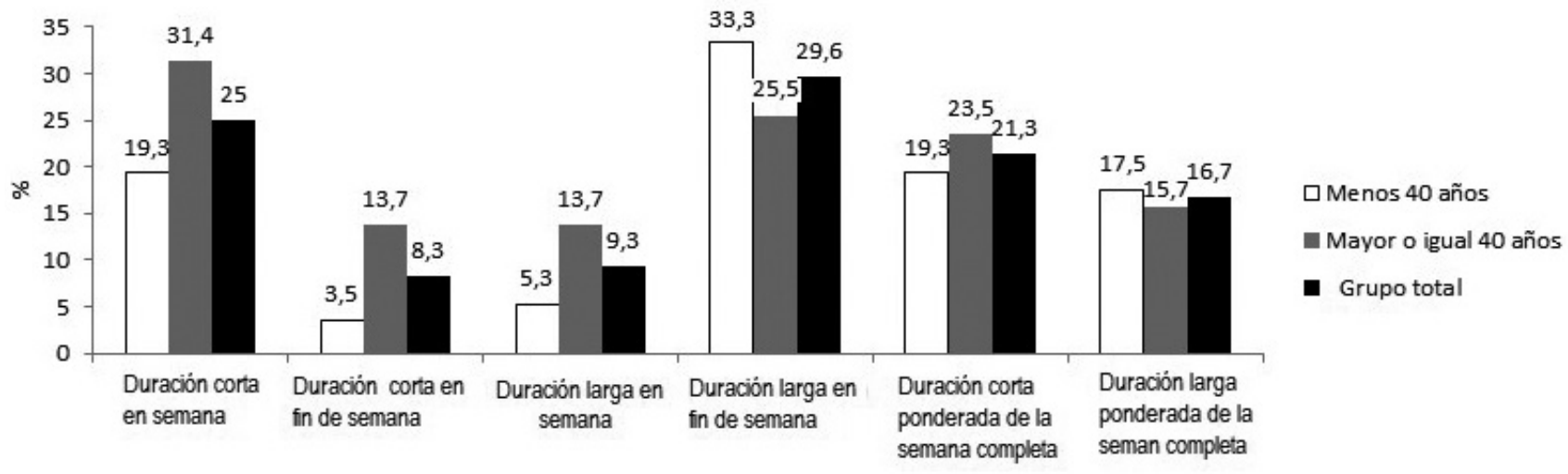

B)

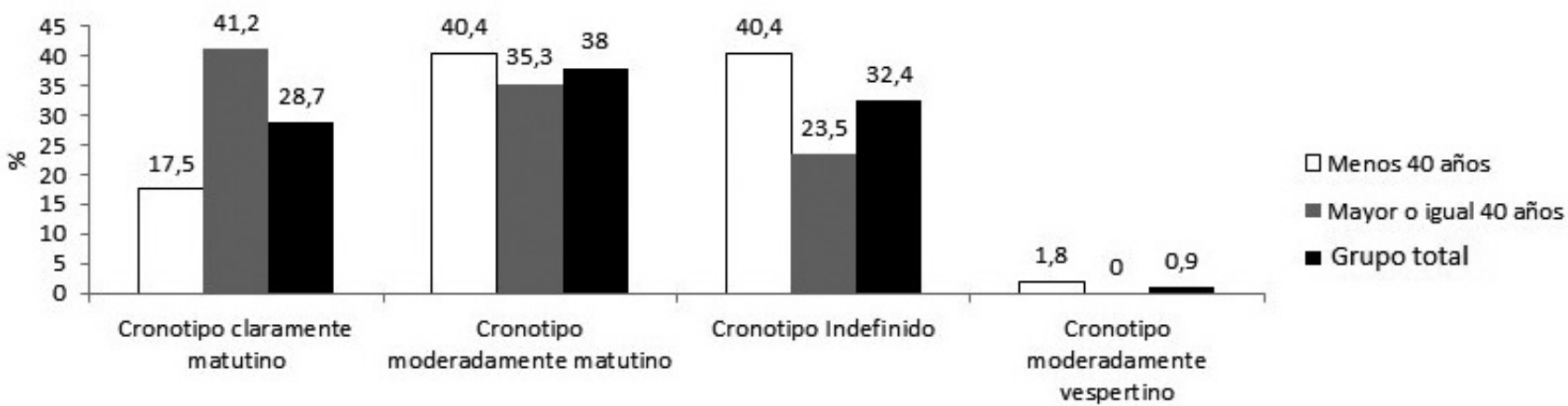

Figura 1. Frecuencia de duración subóptima de sueño (A) y fenotipos circadianos (B) en la muestra total y según grupo etario

Porcentajes calculados con base al número total de mujeres en cada grupo etario 0 grupo total. $n=108$

Por su parte, las mujeres de cronotipo claramente matutino presentaron niveles de presión arterial sistólica $(p=0,018)$ y diastólica $(\mathrm{p}=0,017)$ más elevada respecto de las mujeres de cronotipo indefinido. Adicionalmente, en comparación con las mujeres de cronotipo moderadamente matutino, las mujeres claramente matutinas demostraron niveles significativamente más elevados del índice TGL/HDLc $(p=0,031)$ y de GGT $(p=0,049)$ y tendieron a mostrar mayor presión sistólica y cifras de HDLc menores.

En la tabla 2 se presenta la variación de los puntajes de estatus cardiometabólico de acuerdo a la duración 
de sueño y fenotipos circadianos. Al comparar con las mujeres que durmieron 7 a 9 horas, se encontró que los tres puntajes calculados fueron significativamente mayores entre las mujeres con duración corta del sueño en fin de semana. Además, siMS risk score también se encontró más elevado entre las mujeres que durmieron menos de 7 horas durante los días de semana. Los tres puntajes se encontraron más elevados entre las mujeres de cronotipo claramente matutino, en comparación con las de cronotipo moderadamente matutino; siMS risk score también se encontró más elevado respecto de las mujeres de cronotipo indefinido. No se encontraron diferencias cuando se comparó el cronotipo moderadamente matutino con el indefinido.

Duración subóptima de sueño, cronotipos y perfil cardiometabólico

$\mathrm{Ni}$ la duración subóptima del sueño ni el fenotipo circadiano se asociaron al exceso de peso según IMC o al estado proinflamatorio/protrombótico. No obstante, la obesidad abdominal identificada a través de índice $C C / T$, se asoció a duración corta de sueño en el fin de semana $(\mathrm{p}=0,038)$. El cronotipo y la duración del sueño se asociaron significativamente a la HTA, esta alteración fue más frecuente en mujeres claramente matutinas y las que durmieron menos de 7 horas durante el fin de semana ( $p=0,045$ y $p=0,047$, respectivamente). La duración de sueño (corta o larga) no se asoció a SM, por el contrario, el cronotipo claramente matutino se asoció al SM y al número de componentes individuales de este $(p=0,037$ y $p=0,048$, respectivamente).

La duración corta de sueño en días de semana y fin de semana se asoció a elevado ICMet $(p=0,034$ y $p=0,016$, respectivamente). El cronotipo claramente matutino $(p=0,026)$ y la duración corta de sueño en días de semana $y$ fin de semana ( $p=0,031$ y $p=0,001$, respetivamente) se asociaron a elevado siMS risk score.

Tabla 2. Puntajes de estatus cardiometabólico según duración y fenotipos circadianos

\begin{tabular}{|c|c|c|c|}
\hline Duración de sueño y fenotipos circadianos & ICMet & siMS score & siMS score risk \\
\hline \multicolumn{4}{|l|}{ Duración de sueño en semana } \\
\hline$<7$ horas $(n=27)$ & $1,06(0,74-1,93)$ & $2,43(2,25-3,07)$ & $2,34(1,95-3,78)^{*} a$ \\
\hline $7-9$ horas $(n=71)$ & $0,95(0,58-1,49)$ & $2,31(2,13-2,69)$ & $1,91(1,35-2,74)$ \\
\hline$>9$ horas $(n=10)$ & $1,07(0,62-2,39)$ & $2,38(2,14-3,43)$ & $2,34(1,79-4,31)$ \\
\hline \multicolumn{4}{|l|}{ Duración de sueño en fin de semana } \\
\hline$<7$ horas $(n=9)$ & $1,88(0,90-2,20)$ & $3,07(2,55-3,94)^{*} a$ & $3,98(2,57-4,82)^{*} a$ \\
\hline $7-9$ horas $(n=67)$ & $1,03(0,62-1,54)$ & $2,40(2,16-2,77)$ & $2,06(1,29-2,78)$ \\
\hline$>9$ horas $(n=32)$ & $0,86(0,63-1,69)$ & $2,31(2,14-2,96)$ & $2,07(1,47-3,06)$ \\
\hline \multicolumn{4}{|l|}{ Duración de sueño ponderada } \\
\hline$<7$ horas $(n=23)$ & $1,06(0,74-1,88)$ & $2,40(2,25-3,07)$ & $2,29(1,39-3,78)$ \\
\hline $7-9$ horas $(n=67)$ & $1,02(0,63-1,63)$ & $2,43(2,05-2,79)$ & $2,07(1,35-2,81)$ \\
\hline$>9$ horas $(n=18)$ & $0,86(0,54-1,11)$ & $2,31(2,14-2,49)$ & $2,08(1,52-3,05)$ \\
\hline \multicolumn{4}{|l|}{ Fenotipo circadiano o cronotipo } \\
\hline Claramente matutino $(n=26)$ & $1,16(0,80-1,76)^{*} b$ & $2,70(2,31-3,10)^{* *} b$ & $2,85(2,03-3,55)^{\star *} b, c$ \\
\hline Moderadamente matutino $(n=25)$ & $0,74(0,45-1,21)$ & $2,26(1,91-2,59)$ & $1,77(1,14-2,54)$ \\
\hline Indefinido $(n=26)$ & $0,89(0,73-1,59)$ & $2,39(2,21-2,60)$ & $1,98(1,38-2,62)$ \\
\hline
\end{tabular}

*Datos expresados como mediana (rango intercuartílico). Prueba de $U$ de Mann Whitney entre categorías de sueño o cronotipos. ${ }^{*} \mathrm{p}<0,05 ;{ }^{* *} \mathrm{p}<0,01, \mathrm{a} 7-9 \mathrm{~h}$ vs. < 7h. b Claramente matutino vs. moderadamente matutino. c Claramente matutino vs. indefenido.

El análisis de regresión logística reveló que solo el cronotipo claramente matutino predijo significativamente la presencia de SM (OR (CI95 \%): 4,033 (1,078-15,086); $p=0,038)$. Sin embargo, ni la duración del sueño o el cronotipo fueron predictores de obesidad abdominal o de
HTA. Por otro lado, la elevación de ICMet fue predicha por la duración corta de sueño en días de semana (OR (CI95 \%): 4,607 (1,134-18,712); $p=0,033)$ y fin de semana (OR (Cl95\%): 15,375 (1,954-120,974); $p=0,009)$; mientras que la elevación de siMS risk score fue significativamente 
predicha por la duración corta de sueño en días de semana (OR (Cl95 \%): 4,714 (1,162-19,133); $\mathrm{p}=0,030)$ y fin de semana (OR (CI95\%): 42,000 (3,737-472,058); $p=0,002)$, y también por el cronotipo claramente matutino (OR (C195\%): $10,667(1,222-93,127) ; \quad p=0,032)$. Estas asociaciones desaparecieron cuando se ajustó por edad y condición de posmenopausia.

\section{DISCUSIÓN}

Esta investigación estuvo dirigida, fundamentalmente, a explorar la frecuencia de la duración subóptima de sueño y de los fenotipos circadianos, así como a establecer sus principales correlatos sociodemográficos, de estilo de vida y de riesgo cardiometabólico, en un grupo de mujeres adultas. El sueño y la expresión fenotípica del ritmo circadiano individual a través de los cronotipos ha sido evaluada primordialmente en países desarrollados occidentales de Norteamérica y Europa, y, en menor extensión, en países asiáticos como China. La duración del sueño y la frecuencia de duración subóptima de sueño (corta y larga) fueron similares a lo informado específicamente en sudamericanos residentes de cuatro urbes de Estados Unidos de Norteamérica ${ }^{(25)}$. No obstante, la frecuencia de la duración corta de sueño en nuestro trabajo fue menor a la encontrada en los estudios de cohorte realizados en la población china ${ }^{(26)}$ yestadounidense ${ }^{(27,28)}$ (con representación mayoritaria de individuos blancos no hispánicos), en cuyo caso osciló entre 23 \% y $37 \%$. La duración larga fue mayor a la encontrada en estadounidenses ${ }^{(27,28)}$ pero similar a la observada en chinos ${ }^{(26)}$.

Venezuela está ubicada cerca del ecuador ( $\left.\mathrm{N} 8^{\circ} 0^{\prime} 0^{\prime \prime}\right)$, por lo que es un país de clima predominantemente tropical en el que no tienen lugar grandes cambios estacionales anuales, característica que lo hace diferente de terceros situados en otras latitudes en las cuales se ha evaluado la preferencia circadiana. En la presente investigación resalta una marcada tendencia a la matutinidad del grupo estudiado, con escasa presencia de individuos vespertinos y que desplaza al cronotipo indefinido como la tipología circadiana más frecuentemente observada en Europa y Norteamérica ${ }^{(29,30)}$. Tal resultado está en línea con hallazgos previos ${ }^{(13,31)}$ donde, al evaluar las diferencias en las preferencias circadianas entre diferentes países, se informó mayor orientación hacia la matutinidad en países como Colombia, España e India respecto de Estados Unidos de Norteamérica, Holanda, Alemania y Eslovaquia. Por su parte von Schantz et al. ${ }^{(14)}$, al emplear el MEQ en su versión completa, encontraron que la población de Baependi, un pequeño pueblo del estado de Minas Gerais (Brasil), está fuertemente orientada a la matutinidad, en la que más del $50 \%$ de los individuos estudiados fueron clasificados como claramente matutinos y solo uno como moderadamente vespertino, lo cual difiere de la distribución encontrada en Sao Paulo y Londres, en la que se observa mayor vespertinidad. El ciclo de luz solar/oscuridad generado por los movimientos de rotación y traslación de la Tierra es considerado el principal y más importante sincronizador ambiental del ritmo circadiano. La luz solar alcanza diferencialmente al planeta de acuerdo a la latitud. Un estudio realizado en el sur de Brasil reveló un gradiente latitudinal en los cronotipos según el cual se eleva la vespertinidad a medida que se incrementa la latitud o distancia angular entre la línea ecuatorial y un punto determinado de la Tierra ${ }^{(32)}$.

Adicionalmente, es importante considerar el impacto de la etnicidad/raza sobre el fenotipo circadiano para tratar de explicar la orientación matutina de gran parte de la muestra estudiada. La mayoría de la población venezolana tiene un fondo o background genético mestizo, con raíces indígenas propias del continente americano que se funden con las europeas (principalmente españolas) y afroamericanas provenientes de la colonización. En una muestra de adultos de Reino Unido se observó que el cronotipo matutino (respecto del indefinido) fue más prevalente entre individuos de raza negra ${ }^{(33)}$, mientras que Egan et al. (34) en Baepandi (Brasil) solo demostraron asociación entre la matutinidad y el grado de ascendencia amerindia, no así con la europea o africana. Lo anteriormente expuesto permite sugerir que es necesario continuar las investigaciones centradas en la influencia de la etnicidad sobre los fenotipos circadianos en poblaciones mestizas como la venezolana, sin dejar de recordar que existen múltiples sincronizadores externos (luz artificial, interacción social, ejercicio físico, patrones de alimentación) y factores socioculturales no evaluados que, en interacción, inciden sobre las preferencias circadianas y podrían explicar la orientación matutina observada en este trabajo.

Con el empleo de polisomnografía, un metaanálisis sobre los cambios en los patrones de sueño a lo largo de la vida en el ser humano demostró entre adultos una disminución lineal del tiempo total de sueño con la edad, asimismo la disminución de la eficiencia del sueño fue más evidente a partir de los 40 años y el efecto de la edad sobre el tiempo total de sueño fue mayor entre las mujeres ${ }^{(35)}$. En nuestro estudio, la DSS, DSFS y DSP tendieron a ser menores en las mujeres de $\geq 40$ años, observándose una asociación significativa entre la edad y la duración subóptima del sueño, donde la duración corta del sueño fue más frecuente entre estas mujeres, mientras que la duración larga fue de mayor frecuencia entre las mujeres más jóvenes, aunque la asociación no alcanzó significancia estadística. Trabajos previos han informado hallazgos similares entre mujeres venezolanas ${ }^{(11)}$, chinas ${ }^{(26)}$ y estadounidenses ${ }^{(36)}$.

También se ha demostrado que el cronotipo no solo es dependiente de factores genéticos y ambientales sino también de la edad, se observa que al avanzar esta se 
eleva la matutinidad ${ }^{(37,38)}$, tal como se evidenció en el grupo de mujeres evaluadas. Se ha hipotetizado que este efecto de la edad se debe a que con los años el control homeostático del sueño nocturno pierde fuerza lo que conduce a levantarse en horas más tempranas (38), observación que también se produjo en el presente trabajo. No obstante, cabría plantear una posible interacción de la edad con factores socioculturales asociados con el rol social que cumple la mujer, especialmente en sociedades marcadamente matriarcales como la venezolana, donde la mujer ocupa la posición más elevada dentro de las familias en relación a su responsabilidad como sostén del hogar y proveedora del cuidado de hijos y hasta de nietos que sobrevienen muy pronto, prefiriendo levantarse más temprano para tratar de "alargar o multiplicar" sus horas activas del día a fin de cumplir/conciliar las tareas del hogar y las jornadas de trabajo.

En cuanto a otras variables sociodemográficas y de hábitos de vida, se encontró que el estado civil, la ocupación y la condición de exfumadora fueron asociadas solo a la duración subóptima del sueño. En diversos estudios ${ }^{(26,28,39)}$, divorciadas y solteras presentaron mayor probabilidad de duración corta de sueño, por el contrario, en nuestra muestra mostraron mayor frecuencia de duración larga del sueño, las razones para este contraste no son evidentes, aunque probablemente estén implicados factores de orden sociocultural que no han sido explorados. La ocupación es uno de los factores determinantes del estrato socioeconómico, se reporta que los individuos de bajo estrato tienen mayor dificultad para dormir, menor duración y calidad de sueño, aunque factores como la edad, sexo y diferencias socioculturales pueden modificar tal relación ${ }^{(40)}$. Tal como se observó previamente en mujeres chinas ${ }^{(41)}$, en este trabajo la duración corta de sueño fue más común entre las mujeres con ocupación manual y amas de casa, esta observación se puede atribuir a un mayor nivel de estrés psicosocial generado por la disposición más restringida de recursos económicos y desempeño de cargas de trabajo más pesadas en horarios extendidos. Por último, también se confirmó el efecto deletéreo del hábito tabáquico sobre el sueño que anteriormente ha sido documentado (36), demostrándose en las mujeres exfumadoras mayor frecuencia de duración corta del sueño. La arquitectura del sueño se ve alterada por la acción directa de la nicotina sobre la liberación de neurotransmisores implicados en el ciclo sueño/vigilia, por la abstinencia de la nicotina al cesar el hábito y por el desarrollo de patologías secundarias que afectan el sueño ${ }^{(42)}$.

El cese de la capacidad reproductiva en la mujer trae consigo reducción de las hormonas sexuales femeninas y cambios fisiológicos y psicológicos. Randler y Bausback (43) confirmaron que la etapa de la transición menopáusica, más que la edad cronológica, es predictora de la matutinidad en la mujeres; se observó que las mujeres posmenopáusicas presentaron puntajes de matutinidad mayores que las premenopáusicas, lo que coincide con el hallazgo de esta investigación en la que más del $50 \%$ de las mujeres posmenopáusicas presentaron cronotipo claramente matutino en conjunto con una hora de levantarse más temprana durante los fines de semana entre las mujeres de más de 40 años, lo que indica un avance de la fase del ritmo circadiano.

Dada la alta prevalencia de las enfermedades cardiometabólicas en Venezuela, gran parte de nuestro interés se centró en revisar la asociación entre indicadores de riesgo cardiometabólico con la duración subóptima del sueño. En tal sentido, encontramos pocos correlatos para la duración larga de sueño, solo observamos que las mujeres que informaron dormir más de 9 horas presentaron niveles más elevados de glicemia y de transaminasas respecto de las mujeres que durmieron 7 a 9 horas/noche. Cappuccio et al. ${ }^{(44)}$ demostraron, mediante un metaanálisis, que la duración larga de sueño predice la incidencia de la diabetes tipo 2, mientras Cespedes et al. ${ }^{(45)}$ concluyeron que existe una asociación significativa entre la duración larga de sueño sin insomnio y la prevalencia de diabetes en un grupo de latinos e hispanos residentes en Estados Unidos, luego de ajustar por la presencia de obesidad. Los estudios que exploran la variación de biomarcadores hepáticos con la duración larga de sueño son escasos, los existentes se orientan a probar la asociación entre hígado graso no alcohólico y duración de sueño. En este campo, Imaizumi et al. ${ }^{(46)}$ encontraron que, solo en mujeres, la frecuencia de esta patología hepática siguió una curva en forma de $U$ a través de las categorías de la duración del sueño, y observaron diferencias para TGO, pero no para TGP ni GGT según la duración de sueño. Los mecanismos fisiopatológicos implicados en las observaciones del presente estudio no son evidentes, aunque es probable que la duración larga de sueño sea reflejo de hábitos de estilo de vida poco saludables (ej. baja actividad física y dieta no equilibrada) que se asocian a trastornos metabólicos, síntomas depresivos o a otras comorbilidades subclínicas no diagnosticadas.

La duración corta del sueño exhibió un mayor número de asociaciones con condiciones de elevado RCM. El déficit de sueño se encontró asociado a obesidad abdominal y HTA, así como a la elevación significativa de biomarcadores como el índice TGO/TGP, GGT y ácido úrico. Es probable que el aumento de la relación TGO/ TGP se relacione con la manifestación hepática de la desregulación metabólica que condiciona la privacion de sueño. Por su parte, Kanagasabai y Ardern ${ }^{(47)}$ al estudiar el papel de antioxidantes y marcadores inflamatorios en la relación sueño-salud cardiometabólica, encontraron valores más elevados de ácido úrico y GGT entre individuos con corta y muy corta duración de sueño, además, ambos mediaron la relación de la duración de sueño con el SM, CC y presión sistólica, lo cual se explica por el papel atribuido 
a la inflamación y al estrés oxidativo sobre el desarrollo de las enfermedades cardiometabólicas. Merece especial atención que la duración corta de sueño predijo alto RCM según ICMet y siMS risk score, así las mujeres con déficit de sueño demostraron niveles más altos de los tres puntajes continuos del estatus cardiometabólico que se calcularon. Tales resultados se encuentran en línea con evidencias anteriores que demostraron en individuos con déficit de sueño mayor incidencia de diabetes ${ }^{(44)}$ y riesgo de hipertensión ${ }^{\left({ }^{48}\right)}$, donde las mujeres fueron al parecer más susceptibles a los efectos presores de la insuficiencia del sueño. Al aplicar el IMC, diversos estudios han confirmado una asociación entre duración corta del sueño y obesidad (4); no obstante, nuestro estudio solo halló una asociación significativa con la obesidad abdominal cuando se empleó el índice $\mathrm{CC} / \mathrm{T}$, un indicador que pocas veces se ha utilizado al examinar la relación duración de sueño-masa corporal.

La contribución del déficit de sueño a la fisiopatología de las enfermedades cardiometabólicas y su riesgo entraña diversos mecanismos que se solapan y entrecruzan. Uno de ellos es la activación del eje hormonal del estréssistema inmune que perturba la regulación homeostática en células inmunes, hepatocitos, adipocitos, endoteliales y vasculares, lo que crea un ambiente proinflamatorioprotrombótico, insulinoresistencia y elevación de la presión arterial ${ }^{(49)}$. Asimismo, otros mecanismos serían el incremento del apetito, la disminución del empleo de la glucosa en el cerebro y el incremento de la actividad simpática ${ }^{(5)}$.

Para nuestro conocimiento, el presente trabajo es el primero en analizar en una muestra de mujeres venezolanas los fenotipos circadianos en asociación con el RCM. Si se considera al cronotipo matutino como referencia, existen observaciones que apuntan a que los individuos vespertinos tendrían mayor riesgo a desarrollar enfermedades cardiometabólicas al presentar estilos de vida poco saludables y alteración de diversos biomarcadores metabólicos (10,50,51). Esta investigación no pudo confirmar tales evidencias ya que solamente una mujer estudiada mostró orientación vespertina. No obstante, en vista de la significativa frecuencia de mujeres claramente matutinas, decidimos explorar si la orientación en extremo a la matutinidad condicionó RCM en el grupo estudiado. En efecto, el cronotipo claramente matutino predijo un alto RCM según siMS risk score y se asoció a HTA, SM y al número de componente individuales del SM presentes. Las mujeres claramente matutinas presentaron concentraciones más bajas de HDLC y cifras mayores de presión arterial sistólica y diastólica, índice TGL/HDLc y de GGT. Aunque algo inesperados, estos hallazgos garantizan la necesidad de profundizar el estudio de los fenotipos circadianos en la población venezolana. Adicionalmente, es preciso mencionar que la asociación entre elevado RCM y la duración corta de sueño o cronotipo claramente matutino desapareció cuando se ajustó por edad o condición posmenopáusica. Esto último implica que el avance de la edad medió tal asociación. La influencia de la edad y la posmenopausia sobre el fenotipo circadiano o el sueño ya fue comentada anteriormente. Asimismo, la asociación entre cronotipo y RCM no puede ser explicada a través de diferencias en la duración de sueño, puesto que esta última no se encontró asociada a un cronotipo en particular (datos no mostrados).

La orientación extrema matutina involucra avance de fase del ritmo circadiano, con exposición a la luz en horas más tempranas que el reloj interno identificaría como correspondientes a las horas de sueño o descanso nocturno. Estudios en modelos animales y en humanos apuntan a que tanto el avance como el retraso de la fase, encierran diversas alteraciones fisiológicas y metabólicas que conducirían a mayor RCM. En tal sentido, se ha observado que la desalineación por avance de fase aplana la curva de secreción de cortisol, disminuye la oxidación de proteínas y aumenta las concentraciones de insulina y la oxidación de carbohidratos, aunque no afecta el gasto energético o el sueño ${ }^{(52)}$. Se ha registrado que el avance de fase en ratas produce un incremento de la transcripción en hipotálamo de los péptidos orexígenos proteína r-Agouti y neuropéptido $\mathrm{Y}$, altera la expresión de genes relacionados con el metabolismo en hígado y tejido adiposo blanco y marrón e incrementa la inflamación y el estrés en retículo endoplásmico en el tejido adiposo blanco ${ }^{(53)}$. La exposición de ratones a avance crónico de fase induce ganancia de peso e incremento de la grasa corporal, trigliceridemia y tamaño de los adipocitos, sin incremento de la ingesta dietaria ${ }^{(54)}$. Plano et al. (55) han propuesto que la desalineación circadiana debida a desincronización por comportamiento o exposición a la luz altera vías endocrinas y autónomas que regulan el balance de nutrientes y el uso/almacenamiento de combustibles, lo que reduce la tolerancia a la glucosa postpandrial y el uso de la glucosa, con el consecuente aumento de la glicemia basal, ácidos grasos libres y tejido adiposo. Cuando se establece crónicamente, la desalineación puede conducir a SM y obesidad.

Una de las limitaciones de este estudio es haber realizado la evaluación de la duración del sueño y los cronotipos basada únicamente en datos autoinformados y no mediante métodos objetivos como la polisomnografía o actimetría. De igual manera, no se cuenta con una validación del MEQr y de su escala en la población venezolana. Por último, se trató de un estudio de corte transversal y de tamaño muestral pequeño, por lo que no se pueden generar inferencias causales entre el RCM y el sueño y los cronotipos ni se pueden generalizar los resultados a la población. 
En conclusión, la duración subóptima del sueño y los fenotipos circadianos se asociaron a variables socioedemográficas, clínicas y de riesgo cardiometabólico en un grupo de mujeres adultas. La duración corta de sueño y el cronotipo claramente matutino predijeron elevado riesgo cardiometabólico. Deben realizarse estudios más amplios que confirmen los hallazgos encontrados.

Agradecimientos: A la Agencia Adventista de Desarrollo y Recursos Asistenciales (ADRA), en especial al personal integrante del Departamento de Salud de la Iglesia Adventista del Séptimo Día, Central de Valencia-Carabobo, Venezuela, quienes con gran espíritu de colaboración y servicio facilitaron materialmente la logística y espacio necesario para realizar las sesiones de despistaje previstas. Al Lic. Esleiker Amaya y Lic. Hanne Campbell por su apoyo técnico en la ejecución de las sesiones de despistaje.

\section{REFERENCIAS BIBLIOGRÁFICAS}

1. World Health Organization. Global status report on noncommunicable diseases 2014. Génova: World Health Organization; 2014. Disponible en: http://apps.who.int/iris/ bitstream/10665/148114/1/9789241564854_eng.pdf?ua=1

2. Ministerio para el Poder Popular para la Salud. Anuario de mortalidad 2013. Caracas: MPPS; 2017. Disponible en: https:// www.ovsalud.org/descargas/publicaciones/documentosoficiales/Anuario-Mortalidad-2013.pdf

3. Beccuti G, Pannain S. Sleep and obesity. Curr Opin Clin Nutr Metab Care. 2011 Jul;14(4):402-12.

4. Cappuccio FP, Taggart FM, Kandala NB, Currie A, Peile E, Stranges $S$, et al. Meta-analysis of short sleep duration and obesity in children and adults. Sleep. 2008 May;31(5):619-26.

5. Knutson KL. Sleep duration and cardiometabolic risk: a review of the epidemiologic evidence. Best Pract Res Clin Endocrinol Metab. 2010 Oct;24(5):731-43.

6. Xi B, He D, Zhang M, Xue J, Zhou D. Short sleep duration predicts risk of metabolic syndrome: a systematic review and metaanalysis. Sleep Med Rev. 2014 Aug;18(4):293-7.

7. Gnocchi D, Bruscalupi G. Circadian Rhythms and Hormonal Homeostasis: Pathophysiological Implications. Biology (Basel). 2017 Mar;6(1):10.

8. Levandovski R, Sasso E, Hidalgo MP. Chronotype: a review of the advances, limits and applicability of the main instruments used in the literature to assess human phenotype. Trends Psychiatry Psychother. 2013;35(1):3-11.

9. García-Maldonado G, Sánchez-Juárez IG, Martínez-Salazar GJ, Llanes-Castillo A. Cronobiología: Correlatos básicos y médicos. Rev Med Hosp Gen Méx. 2011 Abr;74(2):108-14.

10. Yu JH, Yun CH, Ahn JH, Suh S, Cho HJ, Lee SK, et al. Evening chronotype is associated with metabolic disorders and body composition in middle-aged adults. J Clin Endocrinol Metab. 2015 Apr 100(4):1494-502.

11. Querales M, Baloa N, Varela I, Ruiz Fernández N. Insuficiencia de sueño o descanso se asocia a elevado riesgo cardiometabólico en mujeres carabobeñas de estrato socioeconómico bajo. Rev Venez Endocrinol Metab. 2012 Oct;10(3):142-51.

12. Bouscoulet LT, Vázquez-García JC, Muiño A, Márquez M, López MV, Montes de oca MM, et al. Prevalence of sleep related symptoms in four Latin American cities. J Clin Sleep Med. 2008;4(6):579-85.

13. Smith CS, Folkard S, Schmieder RA, Parra LF, Spelten E, Almiral $\mathrm{H}$, et al. Investigation of morning-evening orientation in six countries using the preferences scale. Pers Individ Dif. $2002 \mathrm{Apr}$
19;32(6):949-68

14. von Schantz M, Taporoski TP, Horimoto AR, Duarte NE, Vallada $\mathrm{H}$, Krieger JE, et al. Distribution and heritability of diurnal preference (chronotype) in a rural Brazilian family-based cohort, the Baependi study. Sci Rep. 2015 March 18;5:9214.

15. Hirshkowitz M, Whiton K, Albert SM, Alessi C, Bruni O, DonCarlos L, et al. National Sleep Foundation's sleep time duration recommendations: methodology and results summary. Sleep Health. 2015 Mar;1(1):40-3.

16. Adan Puig A, Almirall H. Estandarización de una escala reducida de matutinidad en población española: diferencias individuales. Psicothema. 1990;2(2):137-49.

17. Organización Mundial de la Salud. El Estado Físico: Uso e Interpretación de la Antropometría Ginebra: World Health Organization; 1995. Disponible en: http://apps. who.int/iris/bitstream/handlehttp://apps.who.int/iris/ bitstream/handle/10665/42132/WHO_TRS_854_spa. pdf; jsessionid=6FC4A30C4FE2956CF4B11829C $1 F$ 1FF3B79 ?sequencez $=1$

18. Ashwell M, Hsieh SD. Six reasons why the waist-to-height ratio is a rapid and effective global indicator for health risks of obesity and how its use could simplify the international public health message on obesity. Int J Food Sci Nutr. 2005 Aug;56(5):303-7.

19. Chobanian AV, Bakris GL, Black HR, Cushman WC, Green LA, Izzo $\mathrm{JL} \mathrm{Jr}$, et al. The Seventh Report of the Joint National Committee on Prevention, Detection, Evaluation, and Treatment of High Blood Pressure: The JNC 7 Report. JAMA. 2003 May;289(19):256072.

20. Ingram GIC. The determination of plasma fibrinogen by the clot weight method. Biochem J. 1952 Aug;51(5):583-5.

21. Alberti KG, Eckel RH, Grundy SM, Zimmet PZ, Cleeman JI, Donato KA, et al. Harmonizing the metabolic syndrome: a joint interim statement of the International Diabetes Federation Task Force on Epidemiology and Prevention; National Heart, Lung, and Blood Institute; American Heart Association; World Heart Federation; International Atherosclerosis Society; and International Association for the Study of Obesity. Circulation. 2009 Oct 20;120(16):1640-5.

22. Zhang Y, Zhu CG, Guo YL, Xu RX, Li S, Dong Q, et al. Higher fibrinogen level is independently linked with the presence and severity of new-onset coronary atherosclerosis among Han Chinese population. PLoS One. 2014 Nov 26;9(11):e113460.

23. Wakabayashi I, Daimon T. The "cardiometabolic index" as a new marker determined by adiposity and blood lipids for discrimination of diabetes mellitus. Clin Chim Acta. 2015 Jan 01;438:274-8.

24. Soldatovic I, Vukovic R, Culafic D, Gajic M, Dimitrijevic-Sreckovic V. siMS Score: Simple Method for Quantifying Metabolic Syndrome. PLoS One. 2016;11(1):e0146143.

25. Patel SR, Sotres-Alvarez D, Castañeda SF, Dudley KA, Gallo LC, Hernández R, et al. Social and Health Correlates of Sleep Duration in a US Hispanic Population: Results from the Hispanic Community Health Study/Study of Latinos. Sleep. 2015 Oct 01;38(10):1515-22.

26. Chen Y, Kartsonaki C, Clarke R, Guo Y, Yu C, Bian Z, et al. Characteristics and correlates of sleep duration, daytime napping, snoring and insomnia symptoms among 0.5 million Chinese men and women. Sleep Med. 2018 Apr;44:67-75.

27. Cepeda MS, Stang P, Blacketer C, Kent JM, Wittenberg GM. Clinical Relevance of Sleep Duration: Results from a CrossSectional Analysis Using NHANES. J Clin Sleep Med. 2016 Jun 15;12(6):813-9.

28. Liu Y, Wheaton AG, Chapman DP, Cunningham TJ, Lu H, Croft JB. Prevalence of Healthy Sleep Duration among Adults-United States, 2014. MMWR Morb Mortal Wkly Rep. 2016 Feb 19;65(6):137-41.

29. Malone SK, Patterson F, Lozano A, Hanlon A. Differences in morning-evening type and sleep duration between Black and 
White adults: Results from a propensity-matched UK Biobank sample. Chronobiol Int. 2017;34(6):740-52.

30. Basnet S, Merikanto I, Lahti T, Männistö S, Laatikainen T, Vartiainen $E$, et al. Associations of common noncommunicable medical conditions and chronic diseases with chronotype in a population-based health examination study. Chronobiol Int. 2017;34(4):462-70.

31. Randler C, Prokop P, Sahu S, Haldar P. Cross-cultural comparison of seven morningness and sleep-wake measures from Germany, India and Slovakia. Int J Psychol. 2015 Aug;50(4):279-87.

32. Leocadio-Miguel MA, Louzada FM, Duarte LL, Areas RP, Alam M, Freire MV, et al. Latitudinal cline of chronotype. Sci Rep. 2017 Jul 14;7(1):5437.

33. Malone SK, Patterson F, Lu Y, Lozano A, Hanlon A. Ethnic differences in sleep duration and morning-evening type in a population sample. Chronobiol Int. 2016 Oct 25;33(1):10-21

34. Egan KJ, Campos Santos H, Beijamini F, Duarte NE, Horimoto AR, Taporoski TP, et al. Amerindian (but not African or European) ancestry is significantly associated with diurnal preference within an admixed Brazilian population. Chronobiol Int. 2017;34(2):26972.

35. Ohayon MM, Carskadon MA, Guilleminault C, Vitiello MV. Metaanalysis of quantitative sleep parameters from childhood to old age in healthy individuals: developing normative sleep values across the human lifespan. Sleep. 2004 Nov 01;27(7):1255-73.

36. Krueger PM, Friedman EM. Sleep duration in the United States: a cross-sectional population-based study. Am J Epidemiol. 2009 May 01;169(9):1052-63.

37. Roenneberg T, Kuehnle T, Juda M, Kantermann T, Allebrandt $\mathrm{K}$, Gordijn $M$, et al. Epidemiology of the human circadian clock. Sleep Med Rev. 2007 Dec;11(6):429-38.

38. Díaz-Morales JF, Parra-Robledo Z. Age and Sex Differences in Morningness/Eveningness Along the Life Span: A Cross-Sectional Study in Spain. J Genet Psychol. 2018 Mar-Apr;179(2):71-84

39. Wang S, Li B, Wu Y, Ungvari GS, Ng CH, Fu Y, et al. Relationship of Sleep Duration with Sociodemographic Characteristics, Lifestyle, Mental Health, and Chronic Diseases in a Large Chinese Adult Population. J Clin Sleep Med. 2017 Mar 15;13(3):377-84.

40. Knutson KL. Sociodemographic and cultural determinants of sleep deficiency: implications for cardiometabolic disease risk. Soc Sci Med. 2013 Feb;79:7-15.

41. Tu X, Cai H, Gao YT, Wu X, Ji BT, Yang G, et al. Sleep duration and its correlates in middle-aged and elderly Chinese women: the Shanghai Women's Health Study. Sleep Med. 2012 Oct;13(9):1138 45.

42. Jaehne A, Loessl B, Bárkai Z, Riemann D, Hornyak M. Effects of nicotine on sleep during consumption, withdrawal and replacement therapy. Sleep Med Rev. 2009 Oct;13(5):363-77.

43. Randler C, Bausback V. Morningness-eveningness in women around the transition through menopause and its relationship with climacteric complaints. Biological Rhythm Research. 2010;41(6):415-31.

44. Cappuccio FP, D'Elia L, Strazzullo P, Miller MA. Quantity and quality of sleep and incidence of type 2 diabetes: a systematic review and meta-analysis. Diabetes Care. 2010 Feb;33(2):41420.

45. Cespedes EM, Dudley KA, Sotres-Alvarez D, Zee PC, Daviglus ML, Shah NA, et al. Joint associations of insomnia and sleep duration with prevalent diabetes: The Hispanic Community Health Study/ Study of Latinos (HCHS/SOL). J Diabetes. 2016 May;8(3):387-97.

46. Imaizumi H, Takahashi A, Tanji N, Abe K, Sato Y, Anzai Y, et al. The Association between Sleep Duration and Non-Alcoholic Fatty Liver Disease among Japanese Men and Women. Obes Facts. 2015;8(4):234-42.

47. Kanagasabai $\mathrm{T}$, Ardern $\mathrm{Cl}$. Contribution of Inflammation, Oxidative Stress, and Antioxidants to the Relationship between Sleep Duration and Cardiometabolic Health. Sleep. 2015 Dec 01;38(12):1905-12.

48. Guo X, Zheng L, Wang J, Zhang X, Zhang X, Li J, et al. Epidemiological evidence for the link between sleep duration and high blood pressure: a systematic review and meta-analysis. Sleep Med. 2013 Apr;14(4):324-32.

49. Rangaraj VR, Knutson KL. Association between sleep deficiency and cardiometabolic disease: implications for health disparities. Sleep Med. 2016 Feb;18:19-35.

50. Merikanto I, Lahti T, Puolijoki $H$, Vanhala $M$, Peltonen $M$, Laatikainen $\mathrm{T}$, et al. Associations of chronotype and sleep with cardiovascular diseases and type 2 diabetes. Chronobiol Int. 2013 May;30(4):470-7.

51. Patterson F, Malone SK, Lozano A, Grandner MA, Hanlon AL. Smoking, Screen-Based Sedentary Behavior, and Diet Associated with Habitual Sleep Duration and Chronotype: Data from the UK Biobank. Ann Behav Med. 2016 Oct;50(5):715-26.

52. Gonnissen HK, Rutters F, Mazuy C, Martens EA, Adam TC, Westerterp-Plantenga MS. Effect of a phase advance and phase delay of the 24-h cycle on energy metabolism, appetite, and related hormones. Am J Clin Nutr. 2012 Oct;96(4):689-97.

53. Herrero L, Valcarcel L, da Silva CA, Albert N, Diez-Noguera A, Cambras T, et al. Altered circadian rhythm and metabolic gene profile in rats subjected to advanced light phase shifts. PLoS One. 2015;10(4):e0122570.

54. Casiraghi LP, Alzamendi A, Giovambattista A, Chiesa JJ, Golombek DA. Effects of chronic forced circadian desynchronization on body weight and metabolism in male mice. Physiol Rep. $2016 \mathrm{Apr}$ 28;4(8):e12743.

55. Plano SA, Casiraghi LP, García Moro P, Paladino N, Golombek DA, Chiesa JJ. Circadian and Metabolic Effects of Light: Implications in Weight Homeostasis and Health. Front Neurol. 2017;8:558.

Fuentes de financiamiento:

Este artículo ha sido financiado por los autores.

Conflictos de interés:

Los autores declaran no tener ningún conflicto de interés.

\section{Correspondencia:}

Nelina Ruiz-Fernández

Dirección: Calle Acuario \#88-20, Urb. Trigal Norte, Valencia. Estado Carabobo, Venezuela.

Teléfono: +582418426674

Correo electrónico: nruiz@uc.edu.ve

Recibido: 11 de setiembre de 2018 Evaluado: 02 de octubre de 2018. Aprobado: 29 de noviembre de 2018 .

( ) La revista. Publicado por Universidad de San Martín de Porres, Perú. (cc) ${ }_{\text {в }}$ Licencia de Creative Commons Artículo en acceso abierto bajo términos de Licencia Creative Commons Atribución 4.0 Internacional. (http://creativecommons.org/licenses/by/4.0/)

\section{ORCID iDs}

Nelina Ruiz-Fernández Doris Nobrega Indira Varela Yolima Fernandez Claudia Mendoza Jhon Jesus Gabriel Villalobos Cesar Vega María Yoris Dessire Zamora https: / /orcid.org/0000-0001-6047-4417 https://orcid.org/0000-0001-8113-1683 https://orcid.org/0000-0001-9808-0712 https://orcid.org/0000-0001-7652-1708 https://orcid.org/0000-0002-2068-6666 https://orcid.org/0000-0003-1805-8289 https://orcid.org/0000-0002-0033-6175 https://orcid.org/0000-0001-8830-1606 https://orcid.org/0000-0002-6174-1174 https://orcid.org/0000-0002-6959-1641 